\title{
Diseño óptimo de armaduras empleando optimización con ondas del agua
}

\section{Optimal design of truss structures using water wave optimization}

\author{
DOI: http://dx.doi.org/10.17981/ingecuc.13.2.2017.11
}

Artículo de investigación científica. Fecha de recepción:7 de octubre de 2017. Fecha de aceptación: 8 de noviembre de 2017.

\section{Carlos Millán-Páramo}

Universidad Tecnológica Federal de Paraná. Curitiba (Brasil)

Universidad de Sucre. Sincelejo (Colombia)

carlos.millan@unisucre.edu.co

Para citar este artículo:

C. Millán Páramo, "Diseño óptimo de armaduras empleando optimización con ondas del agua," INGE CUC, vol. 13, no. 2, pp. 102-111, 2017. DOI: http://doi.org/10.17981/ingecuc.13.2.2017.11

\section{Resumen}

Introducción- En los últimos años, la importancia de los aspectos económicos en el campo de las estructuras ha motivado a muchos investigadores a emplear nuevos métodos para minimizar el peso de éstas. El objetivo principal de la optimización estructural (diseño óptimo) es minimizar el peso de las estructuras al tiempo que se satisfacen todos los requerimientos impuestos por los códigos de diseño.

Objetivo- En este estudio, el algoritmo Optimización con ondas del agua (Water Wave Optimization - WWO) es implementado para resolver el problema de optimización estructural de armaduras en $2 \mathrm{D}$ y $3 \mathrm{D}$.

Metodología- El estudio está compuesto por tres fases principales: 1) formulación del problema de optimización estructural; 2) estudio de los fundamentos y parámetros que controlan al algoritmo WWO y 3) evaluación del desempeño del WWO en problemas de optimización de armaduras reportadas en la literatura especializada.

Resultados- Los valores de peso, peso promedio, desviación estándar y número total de análisis ejecutados para converger al diseño óptimo conseguidos con WWO indican que el algoritmo es una buena herramienta para minimizar el peso de armaduras sujetas a restricciones de esfuerzo y desplazamientos.

Conclusiones- Se observó que el algoritmo WWO es eficaz, eficiente y robusto para resolver diversos tipos de problemas con diferentes números de elementos. Además, WWO requiere menor número de análisis para converger al diseño óptimo en comparación con otros algoritmos.

Palabras clave- Optimización con ondas del agua; optimización estructural; armaduras; metaheurística.

\section{Abstract}

Introduction- In recent years, the importance of economic considerations in the field of structures has motivated many researchers to employ new methods for minimizing the weight of the structures. The main goal of the structural optimization is to minimize the weight of structures while satisfying all design requirements imposed by design codes.

Objective- In this study, the Water Wave Optimization (WWO) algorithm is implemented to solve the problem of structural optimization of $2 \mathrm{D}$ and $3 \mathrm{D}$ truss structures.

Methodology-The study is composed of three main phases: 1) formulation of the structural optimization problem; 2) study of the fundamentals and parameters that control the WWO algorithm and 3) evaluate the WWO performance in optimization problems of truss structures reported in the specialized literature.

Results- The values of weight, average weight, standard deviation and the total number of analyses executed to converge to the optimum design obtained with WWO indicate that the algorithm is a good tool to minimize the weight of truss structures subject to stress and displacements constrained.

Conclusions- It was observed that the WWO algorithm is e $\square$ ectively, $\mathrm{e} \square$ ciently and robust to solve different types of problems, with different numbers of elements. Furthermore, WWO requires a lower number of analyses to converge to the optimum design compared to other algorithms

Keywords- Water wave optimization; structural optimization; truss structures; metaheuristic. 


\section{INTRODUCCIÓN}

Los principales objetivos del diseño estructural son garantizar la seguridad de las estructuras y encontrar un diseño con la máxima ganancia. En términos generales, las condiciones de seguridad se definen como restricciones de diseño, mientras que las funciones objetivos que dependen de las variables de diseño se definen como la ganancia máxima. En los últimos años, los algoritmos metaheurísticos inspirados en la naturaleza se han utilizado comúnmente en la optimización de la ingeniería. Estos algoritmos iterativos son muy efectivos para encontrar valores óptimos precisos de desafiantes problemas de ingeniería con múltiples variables y restricciones. Además, los algoritmos metaheurísticos permiten tener en cuenta las limitaciones del diseño al combinar el proceso de optimización con un análisis de ingeniería preciso. Los algoritmos metaheurísticos se pueden agrupar en: i) algoritmos basados en trayectoria o ii) algoritmos basados población. El recocido simulado (Simulated Annealing, SA), método desarrollado por Kirkpatrick [1], es un algoritmo basado en trayectoria, mientras que búsqueda harmónica (Harmony Search, HS) [2], algoritmos genéticos (AG) [3], búsqueda cuckoo (Cuckoo Search, CS) [4], optimización con enjambre de partículas (Particle Swarm Optimziation, PSO) [5], optimización con colonia de hormigas (Ant Colony Optimization, ACO) [6] son todos algoritmos basados en población.

Las armaduras se han optimizado utilizando varios enfoques. Por ejemplo, Erbatur empleó AG para el diseño óptimo de armaduras planas y espaciales con variables continuas y discretas [7]. Schutte y Groenwold utilizaron PSO para el dimensionamiento y optimización del diseño de armaduras [8]. Camp y Bichon emplearon ACO para minimizar el peso total de la estructura sujeta a restricciones de esfuerzo y desplazamiento [9]. Lee y Geem optimizaron el diseño de armaduras bajo condiciones de carga múltiples mediante el uso del algoritmo HS y variables continuas [10], [11]. El algoritmo Big Bang-Big Crunch (BBBC), desarrollado por Erol y Eksin [12], fue empleado en la metodología de diseño óptimo de armaduras espaciales por Camp [13]. Li desarrolló un optimizador de enjambre de partículas heurístico basado en el optimizador de enjambre de partículas con y un esquema de HS; este método se aplicó con éxito al diseño óptimo de armaduras planas y espaciales [14].

Adicionalmente, Perez y Behdinan optimizaron armaduras con PSO mejorado [15]. Lamberti presentó un eficiente algoritmo SA para optimización de tamaño (dimensionamiento) y forma de armaduras [16]. Un algoritmo hibrido de BB-BC fue usado para la optimización de dimensionamiento de armaduras [17]. Kaveh y Talatahari propusieron un método de optimización híbrido combinando los algoritmos PSO, ACO y HS para optimizar armaduras con variables continuas y discretas [18], [19]. Sonmez propuso una metodología de optimización denominada ABC-AP
(Artificial Bee Colony and Adaptive Penalty) con el fin de reducir el peso de armaduras [20]. Degertekin aplicó dos algoritmos HS mejorados para la optimización de armaduras [21]. Degertekin y Hayalioglu aplicaron TLBO (Teaching Learning Based Optimization) para la optimización de dimensionamiento de armaduras [22]. Camp y Farshchin emplearon un TLBO modificado para el diseño óptimo de armaduras [23]. Kaveh desarrolló un algoritmo híbrido llamado HPSSO (Hybrid Particle Swallow Swarm Optimization) y fue puesto a prueba de problemas de minimización de peso de armaduras [24]. Finalmente, diversos investigadores han desarrollado algoritmos metaheurísticos combinados con la teoría del caos para optimizar armaduras [25]-[28].

Recientemente, nuevos algoritmos metaheurísticos también se están desarrollando para mejorar la capacidad de optimización y el comportamiento de convergencia. Por ejemplo, el algoritmo de optimización con ondas de agua (Water Wave Optimization-WWO) que es un método metaheurístico basado en población desarrollado por Zheng [29] y está inspirado en la teoría ondulatoria de aguas. El algoritmo utiliza una serie de mecanismos que lo hacen efectivo al momento de explorar espacios de búsquedas altamente dimensionales, además, es simple y fácil de implementar utilizando poblaciones pequeñas y solo unos pocos parámetros de control.

En el presente estudio, la metaheurística recientemente desarrollada WWO es aplicada en el problema de diseño óptimo (optimización de dimensionamiento) de armaduras planas y espaciales. La eficacia del algoritmo se demostró al resolver 5 problemas clásicos de minimización de peso. Los resultados de optimización indican que WWO es muy competitivo comparado con otros algoritmos metaheurísticos y puede encontrar diseños eficientes.

\section{Metodología}

La metodología desarrollada para el empleo y evaluación del algoritmo WWO en problemas de optimización de armaduras consistió en 3 etapas principales. La primera fase correspondió a la formulación del problema de optimización estructural. La segunda fase fue el estudio de los fundamentos y parámetros que controlan al algoritmo WWO con el objeto de elaborar un código en el paquete computacional Matlab®. La tercera fase fue evaluar el desempeño del WWO en problemas optimización de armaduras reportadas en la literatura especializada.

\section{A. Formulación del problema de optimización estructural}

El principal objetivo en este tipo de problemas de optimización es minimizar las secciones transversales de los miembros de la estructura con el fin de encontrar el peso mínimo de la estructura satisfaciendo simultáneamente las limitaciones que el problema de opti- 
mización impone [30]. Por consiguiente, la formulación matemática de este problema de optimización se describe de la siguiente manera:

$$
\begin{array}{lr}
\text { minimizar: } W(\{x\})=\sum_{i=1}^{n} \gamma_{i} A_{i} L_{i}(x) \\
\text { sujeto a: } & \mathrm{i}=1,2, \ldots, \mathrm{m} \\
\delta_{\min } \leq \delta_{i} \leq \delta_{\max }, & \mathrm{i}=1,2, \ldots, \mathrm{n} \\
\sigma_{\min } \leq \sigma_{i} \leq \sigma_{\max } & \mathrm{i}=1,2, \ldots, \mathrm{n}
\end{array}
$$

donde $W(\{x\})$ es el peso de la estructura; $n$ es el número de miembros que componen la estructura; $m$ es el número de nodos; $\gamma_{i}$ es la densidad del material del miembro $i ; L_{i}$ es la longitud del miembro $i ; A_{i}$ es la sección transversal del miembro $i$ elegida entre $A_{\text {min }}$ y $A_{\max } ; \sigma_{i}$ y $\delta_{i}$ son los esfuerzos en los miembros y deflexiones en los nodos, respectivamente.

\section{B. Optimización con ondas del agua}

(Water Wave Optimization - WWO)

El algoritmo WWO se inspira en los modelos de onda de agua superficial para resolver problemas de optimización. Sin perder la generalidad, supongamos que tenemos un problema de minimización con la función objetivo $f$. En WWO, el espacio de solución $X$ es análogo al área del lecho marino, y la evaluación de la función objetivo de un punto $x \in X$ se mide directamente proporcional por su profundidad del lecho marino: cuanto más corta sea la distancia al nivel del agua inmóvil, menor será el valor de $f(x)$. Cabe señalar que, por analogía, el espacio 3-D del lecho marino se generaliza a un espacio n-dimensional.

Como la mayoría de las otras metaheurísticas, WWO mantiene una población de soluciones, cada una de las cuales es análoga a una onda que tiene una altura (o amplitud) $h \in Z^{+}$y una longitud de onda $\in R^{+}$. Luego de la inicialización, para cada onda, $h$ se establece como una constante $h_{\max }$ y $\lambda$ se establece en 0.5. Durante el proceso de resolución del problema, consideramos tres tipos de operaciones en las ondas: propagación, refracción y ruptura.

\section{1) Propagación}

Cada onda original de la población de ondas puede propagarse solo una vez en cada iteración. Aquí el operador de propagación desplaza la onda original $x$ en cada dimensión $(d)$ para producir una nueva onda propagada $x$ '. La nueva onda está modelada por la siguiente ecuación:

$$
x^{\prime}(d)=x(d)+R \cdot \lambda \cdot L(d)
$$

donde $R$ es un número aleatorio uniformemente distribuido dentro del rango [-1,1], y $L(d)$ es la longitud de la dimensión del espacio de búsqueda. Si la nueva posición está fuera del rango factible, se reiniciará a una posición aleatoria en el rango. Si $f\left(x^{\prime}\right)$ $<f(x)$, reemplaza x por $x$ ' en la población y la altura de la onda $x$ ' se establece en $h_{\max }$. De lo contrario, se mantiene $x$, pero su altura $h$ se reduce en uno.
Después de cada iteración, se calcula la longitud de onda $(\lambda)$ de cada onda $x$ de la siguiente manera:

$$
\lambda=\lambda \cdot a^{-\left(f(x)-f_{\min }+\varepsilon\right) /\left(f_{\max }-f_{\min }+\varepsilon\right)}
$$

donde $f_{\max } \mathrm{y} f_{\min }$ son respectivamente los valores de la función objetivo máximo y mínimo entre la población actual, a es el coeficiente de reducción de la longitud de onda, y $\varepsilon$ es un número positivo muy pequeño para evitar la división por cero. La ecuación (6) asegura que las ondas con mejores valores de función objetivo tengan longitudes de onda más pequeñas $\mathrm{y}$, por lo tanto, se propagan dentro de rangos más pequeños.

\section{2) Ruptura}

La operación de ruptura se realiza solo en una onda $x$ que encuentra una nueva mejor solución (es decir, $x$ se convierte en el nuevo $x_{\text {best }}$ ). Ejecuta una búsqueda local alrededor de $x_{\text {best }}$ usando $k$ ondas solitarias (número aleatorio entre 1 y un número predefinido $k_{\max }$ ) para simular el rompimiento. Cada onda solitaria $x$ ' de $x$ se obtiene adicionando un desplazamiento a la posición original en cada dimensión $(d)$ de la siguiente manera:

$$
x^{\prime}(d)=x(d)+N(0,1) \cdot \beta \cdot L(d)
$$

donde $\beta$ es el coeficiente de ruptura y $N(0,1)$ es un número aleatorio gaussiano con media 0 y desviación estándar 1 . Si ninguna de las ondas solitaria $x^{\prime}$ es mejor que $x, x$ se mantiene; de lo contrario, $x$ es reemplazado por el mejor entre las ondas solitarias.

\section{3) Refracción}

El operador de refracción hace que una onda $x$ cuya altura disminuya a cero aprenda de $x_{\text {best }}$. Esto imita la divergencia de la onda cuando su rayo no es perpendicular al isobático. La posición de la onda por refracción se calcula como:

$$
x^{\prime}(d)=\left(\frac{N\left(x_{\text {best }}(d)+x(d)\right.}{2}, \frac{\left|x_{\text {best }}(d)-x(d)\right|}{2}\right)
$$

donde $x^{*}$ es la mejor solución encontrada hasta el momento y $N(\mu, \sigma)$ es un número aleatorio gaussiano con media $\mu$ y desviación estándar $\sigma$. Es decir, la nueva posición es un número aleatorio situado en la mitad entre la posición original y la mejor posición conocida, y el valor absoluto de su diferencia. Una vez que finaliza la fase de refracción, la altura de la onda $x$ se restablece a su valor máximo $h_{\max } \mathrm{y}$ su longitud de onda es actualizada por:

$$
\lambda^{\prime}=\frac{\lambda f(x)}{f\left(x^{\prime}\right)}
$$

En general, el operador de propagación hace que las ondas con mejor valor de función objetivo busquen pequeñas áreas y las ondas de peor valor de función objetivo exploren grandes áreas; el operador de refrac- 
ción ayuda a las ondas a escapar del estancamiento en un mínimo local y, así, mejora la diversidad de la población y reduce la convergencia prematura; el operador de ruptura permite una búsqueda intensiva en un área (potencialmente) prometedora. La combinación de los tres operadores proporciona al algoritmo un buen equilibrio entre exploración y explotación. El pseudocódigo del algoritmo WWO se presenta a continuación:

\section{Inicializar aleatoriamente una población}

$P$ de $n$ ondas (soluciones);

2 Mientras cumple criterio de parada hacer

3 Para cada $x \in P$ hacer

Propagar $x$ para $x$ ' basado en la ec. (5); if $f\left(x^{\prime}\right)<f(x)$

$$
\text { if } f\left(x^{\prime}\right)<f\left(x_{\text {best }}\right)
$$

Ruptura de $x^{\prime}$ basado en la ec. (7);

Actualizar $x_{\text {best }}$ con $x^{\prime}$;

Reemplazar $x$ por $x$; else

$$
\begin{aligned}
& x \cdot \mathrm{h}=x \cdot \mathrm{h}-1 \\
& \text { if } x \cdot h=0
\end{aligned}
$$

Refractar $x$ al nuevo $x$ 'segun ec. (8) y (9);

Actualizar las longitudes de onda según ec. (6);

15 retorna $x^{*}$ mejor solución encontrada hasta el momento
El algoritmo WWO tiene 5 parámetros de control y son: i) tamaño de población $n$; ii) altura máxima de onda $h_{\max }$; iii) coeficiente de reducción de longitud de onda $\alpha$; iv) el coeficiente de ruptura 6 ; v) el número máximo de ondas solitarias $k_{\max }$. Según Zheng [29], en general, cuanto mayor sea el $h_{\max }$, mayor será el promedio de vida de las ondas; si $h_{\max }$ es pequeño (1 o 2), las ondas serán reemplazadas frecuentemente por nuevas ondas y, por lo tanto, se incrementará la diversidad de la solución. Un a grande hace que el algoritmo explore un área grande, mientras que un a pequeño hace que el algoritmo realice una explotación más intensa. Además, un pequeño valor de $B$ con un gran $k_{\max }$ ayuda a mejorar las mejores soluciones $\mathrm{y}$, por lo tanto, aumenta la velocidad de convergencia.

\section{Problemas numéricos}

Los siguientes 5 problemas clásicos (Fig. 1) fueron utilizados para investigar la eficiencia y eficacia del algoritmo WWO:

- Armadura plana de 10 barras

- Armadura plana de 17 barras

- Armadura plana de 18 barras

- Armadura espacial de 25 barras

- Armadura espacial de 72 barras
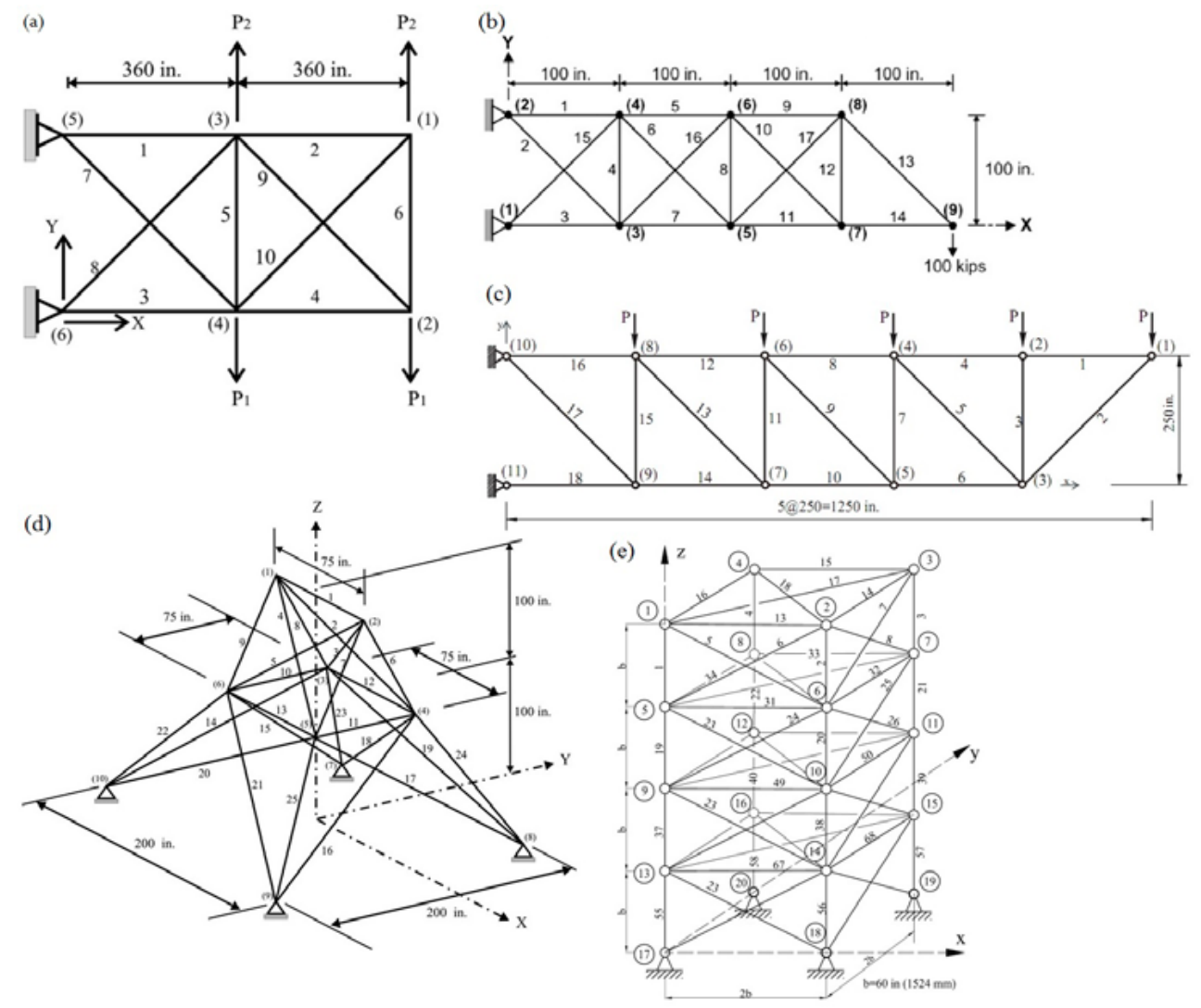

Fig. 1. Armadura de 10 barras (a), 17 barras (b), 18 barras (c), 25 barras (d), 72 barras (e) Fuente: Autores 


\section{1) Armadura plana de 10 barras}

La figura 1a muestra la geometría y las condiciones de carga de la armadura compuesta por 10 barras. En este problema, dos condiciones de cargas fueron consideradas: Caso I: P1 = 100 kips y P2 = 0; Caso II: P1 = 150 kips y P2 $=50$ kips. Los miembros están fabricados de un material con módulo de elasticidad $\mathrm{E}=10000 \mathrm{ksi}$ y densidad por unidad de volumen de $\rho=0,10 \mathrm{lb} / \mathrm{in}^{3}$. El área mínima y máxima para la sección transversal de los miembros es $0,1 \leq A_{i}\left(\mathrm{in}^{2}\right) \leq 35$. Los desplazamientos de los nodos libres no deben exceder \pm 2 in en dirección vertical y horizontal, además, los esfuerzos admisibles tanto a tensión y compresión no deben superar $20 \mathrm{ksi}$. El problema tiene 32 restricciones no lineales (10 restricciones de tensión, 10 restricciones de compresión y 12 restricciones de desplazamiento).

\section{2) Armadura plana de 17 barras}

Este problema tiene 17 variables independientes y 52 restricciones no lineales (17 restricciones de tensión, 17 restricciones de compresión y 18 restricciones de desplazamiento). En la fig. 1b se muestra la geometría y solicitaciones de carga a la que está sometida la estructura. La densidad del material para todos los miembros es de $0,268 \mathrm{lb} / \mathrm{in}^{3}$ y el módulo de elasticidad es de 30000 ksi. El máximo esfuerzo permitido en las barras fue de $\pm 50 \mathrm{ksi}$ con una restricción de desplazamiento en los nodos libres de \pm 2 in en ambas direcciones. La única carga se encuentra en el nodo 9 y es de 100 kips. El área mínima y máxima para la sección transversal de los miembros es $0,1 \leq A_{i}\left(\mathrm{in}^{2}\right) \leq 35$.

\section{3) Armadura plana de 18 barras}

En la fig. 1c se muestra la geometría y condiciones de carga de la armadura de 18 elementos y 11 nodos. El valor de la carga es $\mathrm{P}=20$ kips que actúa en dirección gravitacional en los nodos 1, 2, 4, 6 y 8. Todos los miembros son de un material con módulo de elasticidad $\mathrm{E}=10000 \mathrm{ksi}$ y densidad de 0,10 lb/in ${ }^{3}$. El esfuerzo máximo permitido a tensión y compresión es $20 \mathrm{ksi}$. Adicionalmente, la restricción de esfuerzo al pandeo de Euler debe ser tenida en cuenta para los elementos que se encuentran a compresión. El esfuerzo al pandeo de Euler para el miembro $i$ se calcula como:

$$
\sigma_{i}=-\frac{K E A_{i}}{L_{i}^{2}}
$$

donde $L_{i}$ y $A_{i}$ son la longitud y área de la sección transversal del elemento. $K$ es una constante determinada por la geometría y se le asignó un valor de 4[20]. El número de variables se redujo a cuatro grupos de la siguiente manera: (G1) elementos 1, 4, 8, 12, 16; (G2) elementos 2, 6, 10, 14, 18; (G3) elementos 3, 7, 11, 15; (G4) elementos 5, 9, 13, 17 . El área mínima fue $0.10 \mathrm{in}^{2}$ y la máxima $50 \mathrm{in}^{2}$. El problema tiene 36 restricciones no lineales y sin restricciones de desplazamiento.

\section{4) Armadura espacial de 25 barras}

La torre de transmisión de 25 barras (fig. 1d) se utiliza ampliamente en la optimización estructural para verificar diversas metodologías de diseño y para comparar las diferentes técnicas numéricas. El número de variables para este problema se redujo a 8 grupos de la siguiente manera: (G1) elemento 1; (G2) elementos 2, 3, 4, 5; (G3) elementos 6, 7, 8, 9; (G4) elementos 10, 11; (G5) elementos 12, 13; (G6) elementos 14, 15, 16, 17; (G7) elementos 18, 19, 20, 21; (G8) elementos 22, 23, 24, 25. La densidad del material para todos los miembros es de 0,01 lb/in ${ }^{3}$ y el módulo de elasticidad es de $10000 \mathrm{ksi}$. El máximo esfuerzo permitido en las barras fue de $\pm 40 \mathrm{ksi}$ con una restricción de desplazamiento en los nodos libres (dirección x, y, z) de $\pm 0,35$ in. El área mínima y máxima para la sección transversal de los miembros es 0,01 in $^{2}$ y 3,40 $i^{2}$, respectivamente. La estructura está cargada de acuerdo a Camp [9]. En la fig. 1d se muestra la topología y numeración de nodos para el problema en mención.

\section{5) Armadura en el espacio de 72 barras}

La fig. 1e muestra la numeración de nodos y elementos de la armadura. Para su análisis es categorizada en 16 grupos de variables de diseño. El material tiene un peso por unidad de volumen de $0,10 \mathrm{lb} /$ in $^{3}$ y un módulo de elasticidad de 10000 ksi. Las limitaciones impuestas a la estructura incluyen: un desplazamiento máximo de $\pm 0,25$ in en los nodos superiores en las direcciones $\mathrm{x}, \mathrm{y}$ o $\mathrm{z}, \mathrm{y}$ un esfuerzo máximo admisible de $\pm 25 \mathrm{ksi}$ en cualquier elemento. El rango de áreas de sección transversal aceptables varía de $0,1 \mathrm{in}^{2}$ a 3,0 in ${ }^{2}$. La armadura está sometida a dos condiciones de carga [9]: Caso I, carga en el nodo 17 en las tres direcciones (x, y, z) con valor de 5.0, 5.0 y -5.0 kips; Caso II, carga en los nodos 17, 18, 19 y 20 en la dirección z con valor de -5.0 kips.

\section{Resultados y Discusión}

Una de las ventajas del algoritmo WWO sobre otros métodos metaheurísticos es que el algoritmo tiene pocos parámetros de control. Los resultados numéricos indicaron que con un tamaño de población $n$ de 5 a 10 es adecuado para proporcionar buenos resultados, haciendo que el algoritmo sea más efi- 
ciente desde el punto de vista computacional. Los valores de los demás parámetros fueron: altura máxima de onda $h_{\max }=5$; coeficiente de reducción de longitud de onda $a=1,001$; coeficiente de ruptura $B=0,001$ y el número máximo de ondas solitarias $k_{\max }=\min (12 . \mathrm{D} / 2)$, donde $D$ es la dimensión del problema.

Se realizaron 100 corridas independientes del algoritmo para cada problema. El mejor peso, peso promedio $\left(\mathrm{P}_{\text {prom }}\right)$, desviación estándar (DE) y el número total análisis (NA) para converger al diseño óptimo requeridos en el proceso de optimización encontrados por WWO son comparados con otras técnicas de optimización y son reportados en las tablas. El algoritmo se codificó en el paquete computacional Matlab® y las estructuras se analizaron utilizando el método de la rigidez directa.

\section{A. Armadura plana de 10 barras}

En las Tablas 1 y 2 se presenta una comparación entre los rendimientos de WWO y otras metaheu- rísticas en la optimización de la armadura de 10 barras sujeta a las condiciones de carga del Caso I y II. Los resultados muestran que WWO ha generado diseños más livianos (5060,9 lb para el Caso I y 4677,0 lb para el Caso II) que los reportados por otros algoritmos, solo siendo superados por el reportado en [11], diseño que viola algunas restricciones. Además, la comparación indica que WWO logra estructuras más ligeras en un número menor de análisis y con una desviación estándar más baja.

\section{B. Armadura plana de 17 barras}

El mejor diseño encontrado por WWO fue de 2581,9 lb. En las 100 ejecuciones, el peso promedio fue de 2581,9 lb con una desviación estándar de 0,0lb. Es importante mencionar que los pesos reportados con HS $(2580,8 \mathrm{lb})$ y PSO $(2724,4 \mathrm{lb})$ son menores que los de WWO, esto debido a que estos diseños violan algunas restricciones. En la Tabla 3 se comparan los resultados encontrados en este estudio con los algoritmos mencionados anteriormente.

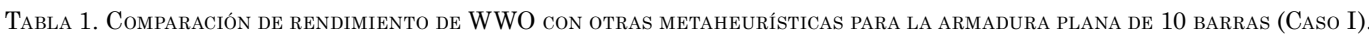

\begin{tabular}{|c|c|c|c|c|c|c|}
\hline Variables (in2) & HS [11] & HPSO [14] & ABC-AP [20] & EHS [21] & TLBO [22] & WWO (este estudio) \\
\hline $\mathrm{A} 1-\mathrm{A} 4$ & 2 & 1,9 & 1,9 & 2 & 1,9 & 1,9 \\
\hline $\mathrm{A} 5-\mathrm{A} 12$ & 0,5 & 0,5 & 0,5 & 0,5 & 0,5 & 0,5 \\
\hline $\mathrm{A} 13-\mathrm{A} 16$ & 0,1 & 0,1 & 0,1 & 0,1 & 0,1 & 0,1 \\
\hline $\mathrm{A} 17-\mathrm{A} 18$ & 0,1 & 0,1 & 0,1 & 0,1 & 0,1 & 0,1 \\
\hline $\mathrm{A} 19-\mathrm{A} 22$ & 1,2 & 1,3 & 1,3 & 1,3 & 1,3 & 1,3 \\
\hline А $23-\mathrm{A} 30$ & 0,5 & 0,5 & 0,5 & 0,5 & 0,5 & 0,5 \\
\hline A31-A34 & 0,1 & 0,1 & 0,1 & 0,1 & 0,1 & 0,1 \\
\hline $\mathrm{A} 35-\mathrm{A} 36$ & 0,1 & 0,1 & 0,1 & 0,1 & 0,1 & 0,1 \\
\hline $\mathrm{A} 37-\mathrm{A} 40$ & 0,5 & 0,5 & 0,5 & 0,5 & 0,5 & 0,5 \\
\hline $\mathrm{A} 41-\mathrm{A} 48$ & 0,5 & 0,5 & 0,5 & 0,5 & 0,5 & 0,5 \\
\hline A49-A52 & 0,1 & 0,1 & 0,1 & 0,1 & 0,1 & 0,1 \\
\hline A53-A54 & 0,2 & 0,1 & 0,1 & 0,1 & 0,1 & 0,1 \\
\hline A55-A58 & 0,2 & 0,2 & 0,2 & 0,2 & 0,2 & 0,2 \\
\hline A59-A66 & 0,5 & 0,5 & 0,5 & 0,6 & 0,5 & 0,5 \\
\hline $\mathrm{A} 67-\mathrm{A} 70$ & 0,5 & 0,4 & 0,4 & 0,4 & 0,4 & 0,4 \\
\hline A71-A72 & 0,6 & 0,6 & 0,6 & 0,6 & 0,6 & 0,6 \\
\hline Peso(lb) & 364,3 & 364,9 & 363,8 & 364,4 & 363,8 & 363,8 \\
\hline Pprom(lb) & - & - & - & 366,8 & 364,4 & 363,9 \\
\hline $\mathrm{DE}(\mathrm{lb})$ & - & - & - & 2 & 0,5 & 0 \\
\hline NA & 20000 & 125000 & 400000 & 13755 & 17954 & 10200 \\
\hline
\end{tabular}

Fuente: Autores 
Tabla 2. Comparación de RENdimiento de WWO con otras metaheurísticas Para la armadura Plana de 10 barras (CASO II).

\begin{tabular}{|c|c|c|c|c|c|c|}
\hline Variables (in2) & HS [11] & HPSO [14] & ABC-AP [20] & EHS [21] & TLBO [22] & WWO (este estudio) \\
\hline A1-A4 & 2 & 1,9 & 1,9 & 2 & 1,9 & 1,9 \\
\hline A5-A12 & 0,5 & 0,5 & 0,5 & 0,5 & 0,5 & 0,5 \\
\hline A13-A16 & 0,1 & 0,1 & 0,1 & 0,1 & 0,1 & 0,1 \\
\hline A17-A18 & 0,1 & 0,1 & 0,1 & 0,1 & 0,1 & 0,1 \\
\hline A19-A22 & 1,2 & 1,3 & 1,3 & 1,3 & 1,3 & 1,3 \\
\hline A23-A30 & 0,5 & 0,5 & 0,5 & 0,5 & 0,5 & 0,5 \\
\hline A31-A34 & 0,1 & 0,1 & 0,1 & 0,1 & 0,1 & 0,1 \\
\hline A35-A36 & 0,1 & 0,1 & 0,1 & 0,1 & 0,1 & 0,1 \\
\hline A37-A40 & 0,5 & 0,5 & 0,5 & 0,5 & 0,5 & 0,5 \\
\hline A41-A48 & 0,5 & 0,5 & 0,5 & 0,5 & 0,5 & 0,5 \\
\hline A49-A52 & 0,1 & 0,1 & 0,1 & 0,1 & 0,1 & 0,1 \\
\hline A53-A54 & 0,2 & 0,1 & 0,1 & 0,1 & 0,1 & 0,1 \\
\hline A55-A58 & 0,2 & 0,2 & 0,2 & 0,2 & 0,2 & 0,2 \\
\hline A59-A66 & 0,5 & 0,5 & 0,5 & 0,6 & 0,5 & 0,5 \\
\hline A67-A70 & 0,5 & 0,4 & 0,4 & 0,4 & 0,4 & 0,4 \\
\hline A71-A72 & 0,6 & 0,6 & 0,6 & 0,6 & 0,6 & 0,6 \\
\hline Peso(lb) & 364,3 & 364,9 & 363,8 & 364,4 & 363,8 & 363,8 \\
\hline Pprom(lb) & - & - & - & 366,8 & 364,4 & 363,9 \\
\hline DE(lb) & - & - & - & 2 & 0,5 & 0 \\
\hline NA & 20000 & 125000 & 400000 & 13755 & 17954 & \\
\hline
\end{tabular}

Fuente: Autores

Tabla 3. Comparación de rendimiento de WWO con otras metaheurísticas para la armadura plana de 17 barras.

\begin{tabular}{|c|c|c|c|c|c|}
\hline Variables (in2) & HS [10] & PSO [14] & PSOPC [14] & HPSO [14] & WWO (este estudio) \\
\hline A1 & 15,8 & 15,8 & 16 & 15,9 & 16 \\
\hline A2 & 0,1 & 2,3 & 0,1 & 0,1 & 12,1 \\
\hline A3 & 12 & 13,9 & 12,1 & 12,1 & 0,1 \\
\hline A4 & 0,1 & 0,1 & 0,1 & 0,1 & 8 \\
\hline A5 & 8,2 & 11,4 & 8,1 & 8,1 & 5,6 \\
\hline A6 & 5,5 & 3,9 & 5,6 & 5,6 & 11,9 \\
\hline A7 & 11,8 & 8,1 & 11,7 & 11,9 & 0,1 \\
\hline A8 & 0,1 & 0,1 & 0,1 & 0,1 & 7,9 \\
\hline A9 & 7,9 & 5,9 & 8 & 8 & 0,1 \\
\hline A10 & 0,1 & 2,3 & 0,1 & 0,1 & 4,1 \\
\hline A11 & 4,1 & 6,3 & 4,1 & 4,1 & 0,1 \\
\hline A12 & 0,1 & 3,4 & 0,1 & 0,1 & 5,6 \\
\hline A13 & 5,7 & 5,4 & 5,7 & 5,7 & 4 \\
\hline A14 & 4,1 & 3,9 & 4 & 4 & 5,6 \\
\hline A15 & 5,7 & 3,5 & 5,6 & 5,5 & 0,1 \\
\hline A16 & 0,1 & 2,3 & 0,1 & 0,1 & 5,6 \\
\hline A17 & 5,6 & 3,5 & 5,6 & 5,5 & 2581,9 \\
\hline Peso (lb) & 2580,8 & 2724,4 & 2582,9 & 2581,9 & 2581,9 \\
\hline Pprom (lb) & - & - & - & - & - \\
\hline DE (lb) & - & - & - & - & 10200 \\
\hline NA & - & - & & & \\
\hline
\end{tabular}

Fuente: Autores 


\section{Armadura plana de 18 barras}

En la Tabla 4 se observa que el mejor diseño encontrado por WWO $(6430,5 \mathrm{lb})$ es igual al reportado por Sonmez [20]. Si bien, el algoritmo HS [11] produce un diseño más ligero $(6421,9 \mathrm{lb})$ que el algoritmo presentado en este estudio, el algoritmo HS viola algunas limitaciones de esfuerzos, mientras que WWO encuentra el peso mínimo sin violar ninguna restricción. Finalmente, la media de los pesos $(6430,5 \mathrm{lb})$ y la desviación estándar (0,0lb) logrados con WWO, muestran la estabilidad que tiene el algoritmo.

Tabla 4. Comparación de Rendimiento de WWO con otras METAHEURÍSTICAS PARA LA ARMADURA PLANA DE 18 BARRAS.

\begin{tabular}{|c|c|c|c|}
\hline Variables & $\begin{array}{c}\text { HS } \\
{[11]}\end{array}$ & $\begin{array}{c}\text { ABC- } \\
\text { AP [20] }\end{array}$ & $\begin{array}{c}\text { WWO } \\
\text { (este estudio) }\end{array}$ \\
\hline A1, A4-A8-A12-A16 (in2) & 10 & 10 & 10 \\
\hline $\begin{array}{c}\text { A2-A6-A10-A14-A18 } \\
\text { (in2) }\end{array}$ & 21,6 & 21,7 & 21,6 \\
\hline A3-A7-A11-A15 (in2) & 12,5 & 12,5 & 12,5 \\
\hline A5-A9-A13-A17 (in2) & 7,1 & 7,1 & 7,1 \\
\hline Peso(lb) & 6421,9 & 6430,5 & 6430,5 \\
\hline Pprom(lb) & - & - & 6430,5 \\
\hline DE(lb) & - & - & 0 \\
\hline NA & 20000 & - & 10200 \\
\hline
\end{tabular}

Fuente: Autores

\section{Armadura espacial de 25 barras}

El mejor diseño encontrado por WWO pesa 545,16 lb (Tabla 5), que es casi igual al mejor resultado citado en la literatura. Se puede observar que WWO es muy competitivo en comparación con las otras técnicas, ya que necesita menor número de análisis para converger al óptimo, además, proporciona un valor de desviación estándar (DE) bajo.

\section{E. Armadura espacial de 72 barras}

Las Tablas 6 y 7 muestran una comparación del diseño de WWO con otras técnicas de optimización. La comparación de los resultados de WWO con los de otros métodos heurísticos muestra que WWO proporciona una mejora en la calidad y consistencia de los diseños de armaduras. Desde el punto de vista estadístico, los resultados constatan la robustez del algoritmo. En línea con los ejemplos anteriores, WWO logra el diseño óptimo después de un número relativamente razonable de análisis estructurales y sin violar ninguna restricción.

\section{Conclusiones}

Se ha conseguido evaluar el desempeño del Algoritmo de Ondas de Aguas (WWO) en el problema de diseño óptimo de armaduras. Cinco problemas clásicos tomados de la literatura fueron analizados para demostrar la eficiencia del algoritmo. Los valores de secciones transversales (áreas), pesos, desviación estándar y número de análisis ejecutados para alcanzar el diseño óptimo fueron comparados con los reportados por otros autores, y mostraron que son coherentes y satisfactorios (Tablas 1, 2, 3, 4, 5, 6, 7), dando así validez al trabajo aquí presentado.

En cuanto a la técnica empleada, se puede observar que WWO tiene eficiencia y eficacia para enfrentar diversos tipos de problemas y con diferentes números de elementos. Esto se ve reflejado en la cantidad de análisis que requirió para alcanzar los diseños óptimos. Este método se puede extender fácilmente para la optimización de otras aplicaciones de diseño estructural.

Tabla 5. Comparación de Rendimiento de WWO CON Otras metaheurísticas Para la armadura espacial de 25 barRas.

\begin{tabular}{|c|c|c|c|c|c|c|c|c|c|c|}
\hline Grupo & ACO [9] & $\begin{array}{c}\text { HPSO } \\
{[14]}\end{array}$ & $\begin{array}{c}\text { BB-BC } \\
{[13]}\end{array}$ & $\begin{array}{c}\text { CMPLSA } \\
{[16]}\end{array}$ & $\begin{array}{c}\text { HBB- } \\
\text { BC }[17]\end{array}$ & $\begin{array}{c}\text { ABC-AP } \\
{[20]}\end{array}$ & $\begin{array}{c}\text { TLBO } \\
{[23]}\end{array}$ & $\begin{array}{c}\text { HPSSO } \\
{[24]}\end{array}$ & $\begin{array}{c}\text { CBO } \\
{[28]}\end{array}$ & $\begin{array}{c}\text { WWO } \\
\text { (este estudio) }\end{array}$ \\
\hline 1 & 0,01 & 0,01 & 0,01 & 0,01 & 0,01 & 0,01 & 0,01 & 0,01 & 0,01 & 0,01 \\
\hline 2 & 2 & 1970 & 2,09 & 1,99 & 1,99 & 1,98 & 1,99 & 1,99 & 2,13 & 1,83 \\
\hline 3 & 2,97 & 3,02 & 2,96 & 2,99 & 3,06 & 3 & 2,99 & 2,99 & 2,89 & 3,18 \\
\hline 4 & 0,01 & 0,01 & 0,01 & 0,01 & 0,01 & 0,01 & 0,01 & 0,01 & 0,01 & 0,01 \\
\hline 5 & 0,01 & 0,01 & 0,01 & 0,01 & 0,01 & 0,01 & 0,01 & 0,01 & 0,01 & 0,01 \\
\hline 6 & 0,69 & 0,69 & 0,69 & 0,68 & 0,67 & 0,69 & 0,68 & 0,68 & 0,68 & 0,7 \\
\hline 7 & 1,68 & 1,68 & 1,6 & 1,68 & 1,64 & 1,68 & 1,68 & 1,68 & 1,61 & 1,73 \\
\hline 8 & 2,67 & 2,64 & 2,69 & 2,66 & 2,68 & 2,65 & 2,66 & 2,67 & 2,69 & 2,57 \\
\hline Peso(lb) & 545,53 & 545,19 & 545,38 & 545,16 & 545,16 & 545,19 & 545,17 & 545,16 & 544,31 & 545,16 \\
\hline Pprom(lb) & 546,34 & - & 545,78 & - & 545,66 & - & 545,48 & - & 545,25 & 545,47 \\
\hline DE(lb) & 0,94 & - & 0,49 & - & 0,367 & - & 0,31 & - & 0,294 & 0,3 \\
\hline NA & 16500 & 125000 & 20566 & - & 12500 & 500000 & 12199 & 13326 & 9090 & 10200 \\
\hline
\end{tabular}

Fuente: Autores 
Tabla 6. Comparación de RENDimiento de WWO con otras metaheurísticas para la armadura espacial de 72 barras (Caso I).

\begin{tabular}{|c|c|c|c|c|c|c|c|c|}
\hline Variables (in2) & ACO [9] & PSO [15] & BB-BC [13] & HBB-BC [17] & TLBO [23] & EHS [21] & TLBO [22] & WWO (este estudio) \\
\hline A1-A4 & 1,9 & 1,7 & 1,9 & 1,9 & 1,9 & 2 & 1,9 & 1,9 \\
\hline A5-A12 & 0,5 & 0,5 & 0,5 & 0,5 & 0,5 & 0,5 & 0,5 & 0,5 \\
\hline A13-A16 & 0,1 & 0,1 & 0,1 & 0,1 & 0,1 & 0,1 & 0,1 & 0,1 \\
\hline A17-A18 & 0,1 & 0,1 & 0,1 & 0,1 & 0,1 & 0,1 & 0,1 & 0,1 \\
\hline A19-A22 & 1,3 & 1,3 & 1,2 & 1,3 & 1,3 & 1,3 & 1,3 & 1,3 \\
\hline A23-A30 & 0,5 & 0,5 & 0,5 & 0,5 & 0,5 & 0,5 & 0,5 & 0,5 \\
\hline A31-A34 & 0,1 & 0,1 & 0,1 & 0,1 & 0,1 & 0,1 & 0,1 & 0,1 \\
\hline A35-A36 & 0,1 & 0,1 & 0,1 & 0,1 & 0,1 & 0,1 & 0,1 & 0,1 \\
\hline A37-A40 & 0,6 & 0,5 & 0,5 & 0,5 & 0,5 & 0,5 & 0,5 & 0,5 \\
\hline A41-A48 & 0,5 & 0,5 & 0,5 & 0,5 & 0,5 & 0,5 & 0,5 & 0,5 \\
\hline A49-A52 & 0,1 & 0,1 & 0,1 & 0,1 & 0,1 & 0,1 & 0,1 & 0,1 \\
\hline A53-A54 & 0,1 & 0,1 & 0,1 & 0,1 & 0,1 & 0,1 & 0,1 & 0,1 \\
\hline A55-A58 & 0,2 & 0,2 & 0,2 & 0,2 & 0,2 & 0,2 & 0,2 & 0,2 \\
\hline A59-A66 & 0,6 & 0,5 & 0,6 & 0,5 & 0,5 & 0,5 & 0,5 & 0,5 \\
\hline A67-A70 & 0,4 & 0,5 & 0,4 & 0,4 & 0,4 & 0,5 & 0,4 & 0,4 \\
\hline A71-A72 & 0,6 & 0,6 & 0,6 & 0,6 & 0,6 & 0,6 & 0,6 & 0,6 \\
\hline Peso(lb) & 380,2 & 381,9 & 379,9 & 379,7 & 379,6 & 381 & 379,6 & 379,6 \\
\hline Pprom(lb) & 383,2 & - & 382,1 & 381,8 & 379,8 & 383,5 & 380,2 & 379,7 \\
\hline DE(1b) & 3,7 & - & 1,9 & 1,2 & 0,1 & 1,9 & 0,4 & 0,2 \\
\hline NA & 18500 & - & 19621 & 13200 & 21542 & 15044 & 19709 & 10200 \\
\hline
\end{tabular}

Fuente: Autores

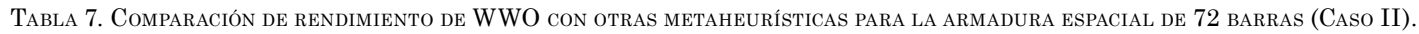

\begin{tabular}{|c|c|c|c|c|c|c|}
\hline Variables (in2) & HS [11] & HPSO [14] & ABC-AP [20] & EHS [21] & TLBO [22] & WWO (este estudio) \\
\hline A1-A4 & 2 & 1,9 & 1,9 & 2 & 1,9 & 1,9 \\
\hline A5-A12 & 0,5 & 0,5 & 0,5 & 0,5 & 0,5 & 0,5 \\
\hline A13-A16 & 0,1 & 0,1 & 0,1 & 0,1 & 0,1 & 0,1 \\
\hline A17-A18 & 0,1 & 0,1 & 0,1 & 0,1 & 0,1 & 0,1 \\
\hline A19-A22 & 1,2 & 1,3 & 1,3 & 1,3 & 1,3 & 1,3 \\
\hline A23-A30 & 0,5 & 0,5 & 0,5 & 0,5 & 0,5 & 0,5 \\
\hline A31-A34 & 0,1 & 0,1 & 0,1 & 0,1 & 0,1 & 0,1 \\
\hline A35-A36 & 0,1 & 0,1 & 0,1 & 0,1 & 0,1 & 0,1 \\
\hline A37-A40 & 0,5 & 0,5 & 0,5 & 0,5 & 0,5 & 0,5 \\
\hline A41-A48 & 0,5 & 0,5 & 0,5 & 0,5 & 0,5 & 0,5 \\
\hline A49-A52 & 0,1 & 0,1 & 0,1 & 0,1 & 0,1 & 0,1 \\
\hline A53-A54 & 0,2 & 0,1 & 0,1 & 0,1 & 0,1 & 0,1 \\
\hline A55-A58 & 0,2 & 0,2 & 0,2 & 0,2 & 0,2 & 0,2 \\
\hline A59-A66 & 0,5 & 0,5 & 0,5 & 0,6 & 0,5 & 0,5 \\
\hline A67-A70 & 0,5 & 0,4 & 0,4 & 0,4 & 0,4 & 0,4 \\
\hline A71-A72 & 0,6 & 0,6 & 0,6 & 0,6 & 0,6 & 0,6 \\
\hline Peso(lb) & 364,3 & 364,9 & 363,8 & 364,4 & 363,8 & 363,8 \\
\hline Pprom(lb) & - & - & - & 366,8 & 364,4 & 363,9 \\
\hline DE(lb) & - & - & - & 2 & 0,5 & 0 \\
\hline NA & 20000 & 125000 & 400000 & 13755 & 17954 & \\
\hline
\end{tabular}

Fuente: Autores 


\section{Financiamiento}

Artículo de investigación derivado del proyecto de investigación titulado "Optimización estructural mediante algoritmos metaheurísticos". Financiado por el grupo de investigación (GRESA) de la Universidad de Sucre. Fecha de inicio: junio de 2016. Fecha de terminación: junio de 2017.

\section{REFERENCIAS}

[1] S. Kirkpatrick, C. D. Gelatt, and M. P. Vecchi, "Optimization by Simulated Annealing," Science 80, vol. 220 , no. 4598 , pp. $671-680,1983$, DOI: $10.1126 /$ science.220.4598.671

[2] Z. W. Geem, J. H. Kim, and G. V. Loganathan, "A New Heuristic Optimization Algorithm: Harmony Search," Simulation, vol. 76 , no. 2, pp. 60-68, 2001, DOI: 10.1177/003754970107600201

[3] J. H. Holland, "Adaptation in Natural and Artificial Systems," Ann Arbor MI Univ. Michigan Press, vol. Ann Ar bor, p. 183, 1975, DOI: 10.1137/1018105.

[4] X.-S. Yang and S. Deb, "Cuckoo search: recent advances and applications," Neural Comput. Appl., vol. 24, no. 1, pp. 169-174, 2014, DOI: 10.1007/s00521-013-1367-1.

[5] J. Kennedy and R. Eberhart, "Particle swarm optimization," 1995 IEEE Int. Conf. Neural Networks (ICNN 95), vol. 4, pp. 1942-1948, 1995, DOI: $10.1109 /$ ICNN.1995.488968.

[6] M. Dorigo, V. Maniezzo, and A. Colorni, "Ant system: optimization by a colony of cooperating agents," IEEE Trans. Syst. Man Cybern. Part B, vol. 26, no. 1, pp. 2941, 1996, DOI: $10.1109 / 3477.484436$.

[7] F. Erbatur, O. Hasançebi, İ. Tütüncü, and H. Kılıç, "Optimal design of planar and space structures with genetic algorithms," Comput. Struct., vol. 75, no. 2, pp. 209-224, 2000, DOI: 10.1016/S0045-7949(99)00084-X.

[8] J. F. Schutte and A. A. Groenwold, "Sizing design of truss structures using particle swarms," Struct. Multi discip. Optim., vol. 25, no. 4, pp. 261-269, oct. 2003, DOI: 10.1007/s00158-003-0316-5.

[9] C. V. Camp and B. J. Bichon, "Design of Space Trusses Using Ant Colony Optimization," J. Struct. Eng., vol. 130, no. 5, pp. 741-751, 2004, DOI: 10.1061/(ASCE)07339445(2004)130:5(741)

[10] K. S. Lee and Z. W. Geem, "A new structural optimization method based on the harmony search algorithm," Comput. Struct., vol. 82, no. 9-10, pp. 781-798, 2004, DOI: $10.1016 /$ j.compstruc.2004.01.002.

[11] K. S. Lee and Z. W. Geem, "A new meta-heuristic algorithm for continuous engineering optimization: Harmony search theory and practice," Comput. Methods Appl. Mech. Eng., vol. 194, no. 36-38, pp. 3902-3933, 2005, DOI: $10.1016 /$ j.cma.2004.09.007.

[12] O. K. Erol and I. Eksin, "A new optimization method: Big Bang-Big Crunch," Adv. Eng. Softw., vol. 37, no. 2, pp. 106-111, 2006, DOI: 10.1016/j. advengsoft.2005.04.005.

[13] C. V. Camp, "Design of Space Trusses Using Big BangBig Crunch Optimization,” J. Struct. Eng., vol. 133, no. 7 , pp. 999-1008, 2007, DOI: 10.1061/(ASCE)07339445(2007)133:7(999).

[14] L. J. Li, Z. B. Huang, F. Liu, and Q. H. Wu, "A heuristic particle swarm optimizer for optimization of pin connected structures," Comput. Struct., vol. 85, no. 7-8, pp. 340-349, 2007, DOI: 10.1016/j.compstruc.2006.11.020.
[15] R. E. Perez and K. Behdinan, "Particle swarm approach for structural design optimization," Comput. Struct., vol. 85 , no. $19-20$, pp. $1579-1588$, 2007, DOI: $10.1016 /$ j. compstruc.2006.10.013.

[16] L. Lamberti, "An efficient simulated annealing algorithm for design optimization of truss structures," Comput. Struct., vol. 86, no. 19-20, pp. 1936-1953, 2008, DOI: $10.1016 /$ j.compstruc.2008.02.004.

[17] A. Kaveh and S. Talatahari, "Size optimization of space trusses using Big Bang-Big Crunch algorithm," Comput. Struct., vol. 87, no. 17-18, pp. 1129-1140, 2009, DOI: 10.1016/j.compstruc.2009.04.011.

[18] A. Kaveh and S. Talatahari, "Particle swarm optimizer, ant colony strategy and harmony search scheme hybridized for optimization of truss structures," Comput. Struct., vol. 87, no. 5-6, pp. 267-283, 2009, DOI: 10.1016/j.compstruc.2009.01.003.

[19] A. Kaveh and S. Talatahari, "A particle swarm ant colony optimization for truss structures with discrete variables," J. Constr. Steel Res., vol. 65, no. 8-9, pp. 1558-1568, 2009, DOI: 10.1016/j.jcsr.2009.04.021.

[20] M. Sonmez, "Artificial Bee Colony algorithm for optimization of truss structures," Appl. Soft Comput., vol. 11, no. 2, pp. 2406-2418, 2011, DOI: 10.1016/j. asoc.2010.09.003.

[21] S. O. Degertekin, "Improved harmony search algorithms for sizing optimization of truss structures," Comput. Struct., vol. 92-93, pp. 229-241, 2012, DOI: 10.1016/j.compstruc. 2011.10.022.

[22] S. O. Degertekin and M. S. Hayalioglu, "Sizing truss structures using teaching-learning-based optimization," Comput. Struct., vol. 119, pp. 177-188, 2013, DOI: 10.1016/j.compstruc.2012.12.011.

[23] C. V. Camp and M. Farshchin, "Design of space trusses using modified teaching-learning based optimization," Eng. Struct., vol. 62-63, pp. 87-97, 2014, DOI: 10.1016/j.engstruct.2014.01.020.

[24] A. Kaveh, T. Bakhshpoori, and E. Afshari, "An efficient hybrid Particle Swarm and Swallow Swarm Optimization algorithm," Comput. Struct., vol. 143, pp. 40-59, 2014, DOI: 10.1016/j.compstruc.2014.07.012.

[25] A. Kaveh and M. Ilchi Ghazaan, "Enhanced colliding bodies optimization for design problems with continuous and discrete variables," Adv. Eng. Softw., vol. 77, pp. 66-75, 2014, DOI: 10.1016/j.advengsoft.2014.08.003.

[26] A. Kaveh, R. Sheikholeslami, S. Talatahari, and M. Keshvari-Ilkhichi, "Chaotic swarming of particles: A new method for size optimization of truss structures," Adv. Eng. Softw., vol. 67, pp. 136-147, 2014, DOI: 10.1016/j.advengsoft.2013.09.006.

[27] A. Kaveh, B. Mirzaei, and A. Jafarvand, "An improved magnetic charged system search for optimization of truss structures with continuous and discrete variables," Appl. Soft Comput. J., vol. 28, pp. 400-410, 2015, DOI: $10.1016 /$ j.asoc.2014.11.056.

[28] A. Kaveh and V. R. Mahdavi, "Colliding Bodies Optimization method for optimum design of truss structures with continuous variables," Adv. Eng. Softw., vol. 70, pp. 1-12, 2014, DOI: 10.1016/j.advengsoft.2014.01.002.

[29] Y.-J. Zheng, "Water wave optimization: A new natureinspired metaheuristic," Comput. Oper. Res., vol. 55, pp. 1-11, 2015, DOI: 10.1016/j.cor.2014.10.008.

[30] C. Millán Páramo and E. Millán Romero, "Algoritmo simulated annealing modificado para minimizar peso en cerchas planas con variables discretas," INGE $C U C$, vol. 12 , no. 2, pp. 9-16, 2016, DOI: $10.17981 /$ ingecuc.12.2.2016.01. 